\title{
Oncofertility in adult and pediatric populations: options and barriers
}

\author{
Joshua A. Halpern, Arighno Das, Cory A. Faw, Robert E. Brannigan \\ Department of Urology, Northwestern University Feinberg School of Medicine, Chicago, IL, USA \\ Contributions: (I) Conception and design: JA Halpern, RE Brannigan; (II) Administrative support: RE Brannigan; (III) Provision of study materials \\ or patients: None; (IV) Collection and assembly of data: A Das, CA Faw; (V) Data analysis and interpretation: JA Halpern, A Das, CA Faw; (VI) \\ Manuscript writing: All authors; (VII) Final approval of manuscript: All authors. \\ Correspondence to: Dr. Robert E. Brannigan. 675 North St. Clair Street, Suite 20-150, Chicago, IL 60611, USA. Email: r-brannigan@northwestern.edu.
}

\begin{abstract}
Cancer and its treatments can affect fertility in a variety of ways, and recent advances in cancer detection and treatment have led to an increasing number of cancer survivors for whom future fertility is a primary concern. Oncofertility is the study of interactions between cancer, anti-cancer therapy, fertility, and reproductive health. Fertility preservation aims to optimize fertility potential before initiation of gonadotoxic therapies. Sperm cryopreservation from an ejaculated sample is the gold standard for adults and post-pubertal adolescents, though added maneuvers such as medical therapy, penile vibratory stimulation, and electroejaculation can be employed when appropriate. When all these approaches fail, testicular sperm extraction can be used to obtain and cryopreserve testicular sperm from the azoospermic patient. Fertility preservation in the pre-pubertal pediatric patient is still experimental, but recent scientific breakthroughs with use of spermatogonial stem cells and testicular tissue transplantation offer great promise for the future. While there may be several practical, cultural, religious, and other barriers to fertility preservation, the establishment of a dedicated fertility preservation team can help to overcome these obstacles and optimize the utilization of fertility preservation in cancer patients of all ages.
\end{abstract}

Keywords: Azoospermia; fertility preservation; oncofertility

Submitted May 08, 2019. Accepted for publication Sep 07, 2019.

doi: $10.21037 /$ tau.2019.09.27

View this article at: http://dx.doi.org/10.21037/tau.2019.09.27

\section{Introduction}

Fifty percent of men will receive a diagnosis of cancer during their lifetime, making cancer among the most common disease states. While the overall incidence of cancer in men is declining, significant advances in early detection and therapy have contributed to increasing numbers of cancer survivors who may have reduced fertility and sexual function (1). Moreover, the improved efficacy of novel cancer therapies is often accompanied by undesirable effects on fertility and sexual function (2). Combined with the increasing age of childbearing, these factors have led to an increase in the cohort of male cancer survivors for whom fertility preservation is an important consideration (3).

A growing body of evidence has shown that fertility potential and reproductive health are among the most important concerns for cancer survivors. According to Lehmann et al., over two-thirds of childhood cancer survivors reported that they would feel distressed if their parenthood goals remained unfilled (4). Other studies have demonstrated that patients with infertility secondary to cancer treatment have increased risk of emotional distress (5-9), and infertility in this setting is associated with significant long-term effects on quality of life (10). For young cancer patients, post-malignancy marriage and parenthood considerations are often considered equally important as treatment of the underlying malignancy and can play a significant role in treatment decisions $(2,11)$.

Oncofertility is the study of interactions between cancer, anti-cancer therapy, fertility, and reproductive health. 
The field of oncofertility arose from the reproductive concerns of cancer patients to meet their unique medical and psychosocial needs. Fertility preservation is a vital component of oncofertility, which employs medical, surgical, and laboratory interventions to preserve reproductive potential in cancer patients before the detrimental effects of cancer and its treatments occur.

Herein we review the effects of cancer and cancer treatment on fertility, options for fertility preservation in male adult and pediatric patients, barriers to care, and the role of a resolute fertility preservation team in overcoming those barriers.

\section{Effects of cancer on fertility}

Cancer is a systemic illness that can affect fertility through multiple distinct mechanisms. Malignancy can alter normal endocrine physiology, which is essential for spermatogenesis. Inflammatory processes and constitutional symptoms can exert both direct and indirect impacts upon spermatogenesis. Psychological morbidity such as anxiety and depression can suppress spermatogenesis and impair sexual function.

Spermatogenesis is dependent upon the normal endocrine function of the hypothalamic-pituitary-gonadal (HPG) axis. Disruption of the axis at any step can result in the suppression of endogenous testosterone production and impaired spermatogenesis. A variety of malignancies may alter the HPG axis, particularly testicular cancers that can produce beta-human chorionic gonadotropin $(\beta-h C G)$ and alpha-fetoprotein (AFP). Elevated levels of $\beta$-hCG are associated with poorer semen quality in patients with testicular cancer, suggesting it plays an inhibitory role in spermatogenesis through negative feedback (12-14). Similarly, elevated levels of AFP have been correlated with decreased sperm count and inhibition of spermatogenesis $(14,15)$.

Beyond the endocrinopathy effects of cancer, the immunological response to malignancy may also affect fertility. Many tumors generate lymphocytic infiltration, which can lead to elevation of pro-inflammatory cytokines (16). Rat models have demonstrated that altered homeostasis of pro-inflammatory cytokines such as IL-6, IL-8, and TNF- $\alpha$ impair the blood-testis barrier, thereby exposing spermatozoa to elevated levels of reactive oxygen species (ROS) that can cause germ cell apoptosis and sloughing (17-20). Anti-sperm antibodies, found predominantly in testicular cancer, may further impair spermatogenesis, though the evidence is equivocal $(21,22)$.

Broader systemic effects of malignancy may exert a negative impact upon reproductive potential in a variety of ways. Cancer-induced anorexia-cachexia syndrome can result in severe malnutrition and, in turn, impaired spermatogenesis (23-25). A simple fever can impair sperm motility, morphology, and concentration, particularly in patients with Hodgkin's lymphoma (26-28). The psychological morbidity of anxiety and depression that often accompanies a diagnosis of malignancy can be associated with decreased testosterone, elevated follicle-stimulating hormone (FSH), and abnormal semen parameters (29). Likewise, the decline in sexual function due to psychological or physiological mechanisms may have an indirect impact on fertility $(30,31)$.

\section{Effects of cancer treatment on fertility}

Treatment of malignancy with chemotherapy, surgery, or radiotherapy can compromise fertility. This may occur through a direct spermatotoxic effect upon germ cells, alteration of the HPG axis, impairment of sexual physiology, or a variety of other mechanisms.

Because chemotherapeutic agents target rapidly dividing cells, spermatozoa are particularly susceptible to the cytotoxic effects of these drugs. Through disruption of DNA synthesis and replication, alkylating agents such as cyclophosphamide and busulfan are the most spermatotoxic chemotherapeutic agents, impairing fertility in up to $60 \%$ of patients (32-34). Effects on spermatogenesis are often irreversible, as approximately $68 \%$ of these patients remain azoospermic 20 years after cessation of therapy (35). Green $e t a l$. developed the cyclophosphamide equivalent dose (CED), a metric for quantification of future risk of infertility, which may help patients and clinicians when devising a treatment regimen (36). However, the authors found that even at low doses, some patients developed azoospermia; as such, there are no definitively "safe" doses of cyclophosphamide. Platinum-based agents, such as cisplatin and carboplatin, are similarly gonadotoxic (37). However, platinum-based agents are associated with more favorable recovery of spermatogenesis over time, as approximately $80 \%$ of patients had successful sperm retrieval within 8 years of cisplatin cessation (38-40). Other chemotherapeutic agents such as antimetabolites and vinca alkaloids exert a more blunted impact upon spermatogenesis (41-44).

Radiation therapy can have a detrimental effect on spermatogenesis, as the immature cell types in the 
testis make it among the most radiosensitive organs. The gonadotoxic effect of radiation is dependent on several variables, including the field of treatment, total dose, and fractionation schedule. There is a clear dosedependent relationship concerning radiotherapy and gonadotoxicity, yet even low doses of less than 0.8 Gy can cause oligozoospermia and doses above 2 Gy can result in irreversible azoospermia.

Surgical management of cancer can have a wide range of negative effects on sexual function and fertility potential. Retroperitoneal and radical pelvic surgery may disrupt branches of the pudendal nerve, lumbar sympathetic, pelvic, and hypogastric plexuses, which are essential for normal erectile and ejaculatory function (45-48). During retroperitoneal lymph node dissection (RPLND) for testis cancer, the postganglionic sympathetic nerves and hypogastric plexus overlying the aorta may be jeopardized, resulting in anejaculation or retrograde ejaculation in up to $89 \%$ of patients (49). While the nerve-sparing approach has led to outstanding improvements in ejaculatory function with $99 \%$ of chemotherapy-naive RPLND patients reporting antegrade ejaculation, functional outcomes are decreased with post-chemotherapy RPLND, wherein 89\% of men report antegrade ejaculation (46). Likewise, men who undergo radical surgery for non-testicular malignancies such as muscle-invasive bladder cancer, retroperitoneal sarcoma, paratesticular rhabdomyosarcoma, and colorectal cancer will often develop transient or permanent ejaculatory and erectile dysfunction despite modern techniques for preservation of sexual function (50-54). Beyond sexual function, men with testis cancer who undergo unilateral orchiectomy may have a decrease in semen parameters from the surgery itself, though the majority of these men will recover normal spermatogenesis if no further treatment is needed (55).

\section{Options for fertility preservation}

Multiple professional societies have issued guidelines and statements about the importance of fertility preservation and options for management in cancer patients (Table 1). All agree that healthcare providers should discuss the possibility of infertility with all male patients newly diagnosed with cancer (Figure 1). The initial discussion should take place soon after diagnosis and prior to initiation of therapy (56). All physicians who care for cancer patients must be comfortable initiating a conversation regarding fertility preservation, as physician recommendations strongly influence a patient's decision to pursue sperm cryopreservation (8).

The gold-standard approach to fertility preservation in adult men is sperm cryopreservation. Modern assisted reproductive technologies including in vitro fertilization (IVF) with intracytoplasmic sperm injection (ICSI) have enabled couples to achieve pregnancy with just a single sperm and a single oocyte. Fertility preservation patients should be counseled about the requirement of IVF-ICSI for any future use of cryopreserved sperm. Furthermore, patients should be informed regarding the harmful effects of the cryopreservation process, which can lead to decreased sperm concentration, motility, and DNA integrity $(65,66)$. Nonetheless, prior studies have shown equivalent pregnancy rates after ICSI using fresh versus cryopreserved ejaculated spermatozoa, and multiple studies have demonstrated success with IVF-ICSI in the setting of male fertility preservation $(67,68)$.

When possible, men presenting for fertility preservation should undergo semen analysis via masturbation. This approach to specimen collection is cost-effective and easily performed in an outpatient or inpatient setting. Avoiding potentially spermatotoxic exposures such as wet heat (e.g., sauna, hot tub) prior to sperm banking, and maintaining a period of abstinence for 2-3 days before collection is recommended (69). Men with sperm in the ejaculate should continue with cryopreservation. While the ideal quantity of cryopreserved sperm is unknown, patients should aim to cryopreserve multiple vials to ensure enough specimen is available for future use. In our practice, we encourage patients to cryopreserve 10-12 vials, when possible. Depending upon a patient's semen parameters, this may require repeated specimen collections in a brief time period.

Erectile dysfunction is prevalent among men with cancer and can hinder the ability to provide an adequate specimen (70). Treatment of erectile dysfunction in the setting of fertility preservation should follow standard treatment paradigms (71). First-line therapy consists of phosphodiesterase-5 (PDE-5) inhibitors followed by intracavernosal injections, both of which supply fast-acting therapy. Other standard treatments for erectile dysfunction such as a medicated urethral system for erections (MUSE) and vacuum erection devices may be tried but can be difficult to access, cumbersome, and less effective in timesensitive scenarios.

In some men, collection via masturbation is not possible due to retrograde ejaculation or anejaculation resulting in aspermia or dry ejaculate. Men with aspermia who can achieve orgasm should undergo post-ejaculate urinalysis 
Table 1 Summary of male fertility preservation guidelines (adapted with permission from Halpern et al., "Guideline based approach to male fertility preservation", Urologic Oncology 2019)

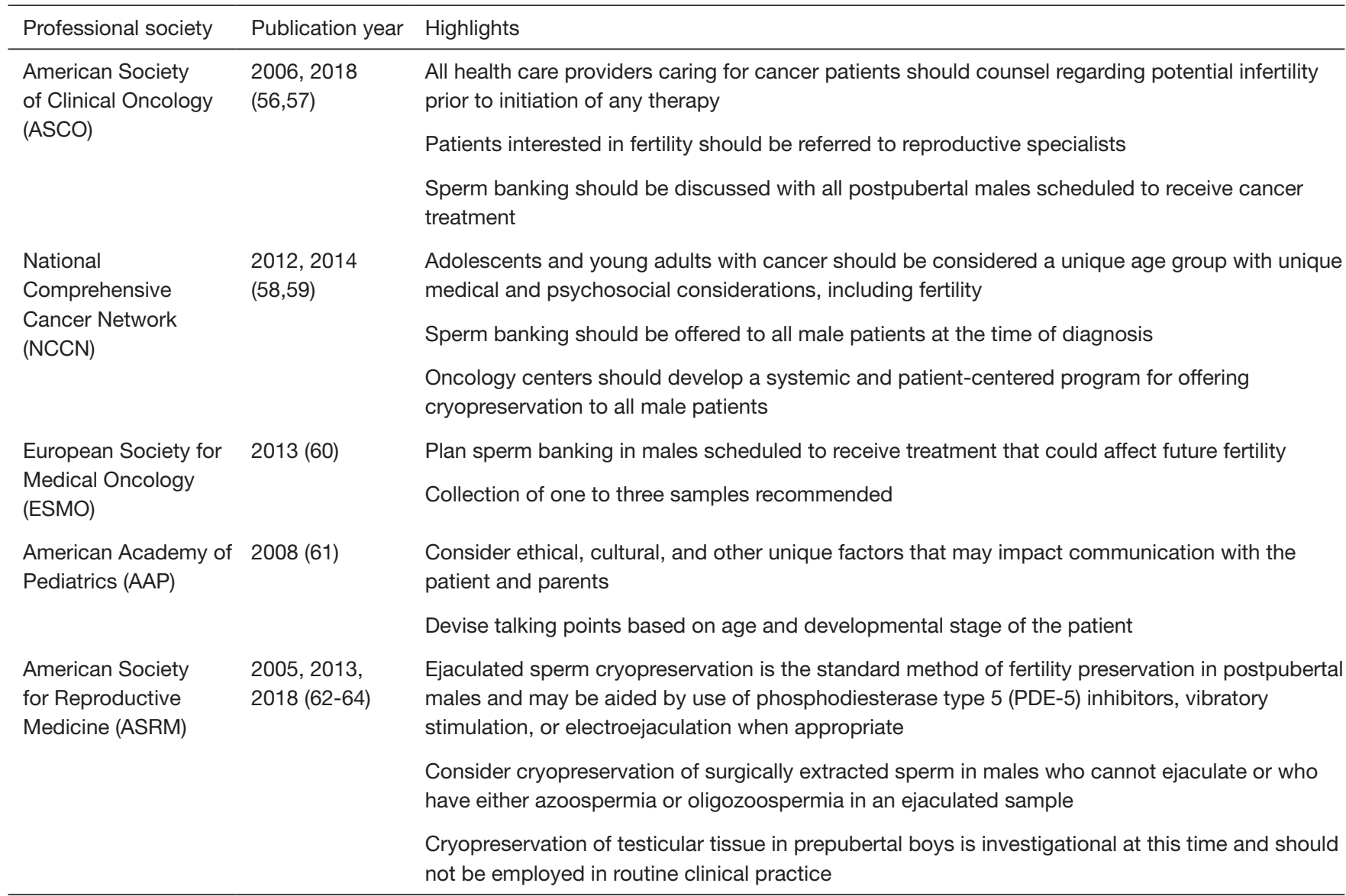

(PEU) to rule out retrograde ejaculation. Sperm found in the PEU indicates retrograde ejaculation, and medical therapy with alpha-agonists such as pseudoephedrine and midodrine or tricyclic antidepressants such as imipramine may be used in an off-label fashion to help restore antegrade ejaculation (72). Shoshany et al. reported improved semen parameters in $70 \%$ of patients treated with pseudoephedrine for retrograde ejaculation (73). In patients unresponsive to medical therapy or when time constraints limit, urinary sperm retrieval can be attempted following urine alkalization, though pregnancy rates using this procedure are highly variable ranging from $20 \%$ to $50 \%(72,74,75)$.

Anejaculation is the complete lack of ejaculation and is accompanied by lack of antegrade release of semen from the urethra. It can result from a variety of conditions, including hypogonadism, neurologic impairment, and medication side effects. While some men will respond to alpha-agonist therapy, many will require added intervention such as penile vibratory stimulation (PVS) and electroejaculation (EEJ).

PVS employs mechanical stimulation of the dorsum and frenulum of the penis, which stimulates the sacral spinal ejaculatory reflex until antegrade ejaculation occurs. Success rates range from $77 \%$ to $86 \%$ in men with spinal cord injury above T10 in whom the ejaculatory reflex is still intact. In men with lower spinal cord injury, the ejaculatory reflex is typically compromised, and success rates are lower at approximately $17 \%$ (76). Advantages of PVS include ease of use, low cost, and low morbidity. As such, PVS should be considered as the first choice in men with anejaculation who are refractory to medical therapy.

EEJ is recommended for patients in whom PVS does not restore antegrade ejaculation. EEJ utilizes a transrectal probe for direct transmission of electrical current to the prostate and seminal vesicles resulting in ejaculation with approximately $95 \%$ success (77). In combination with assisted reproductive technology (ART), EEJ offers 


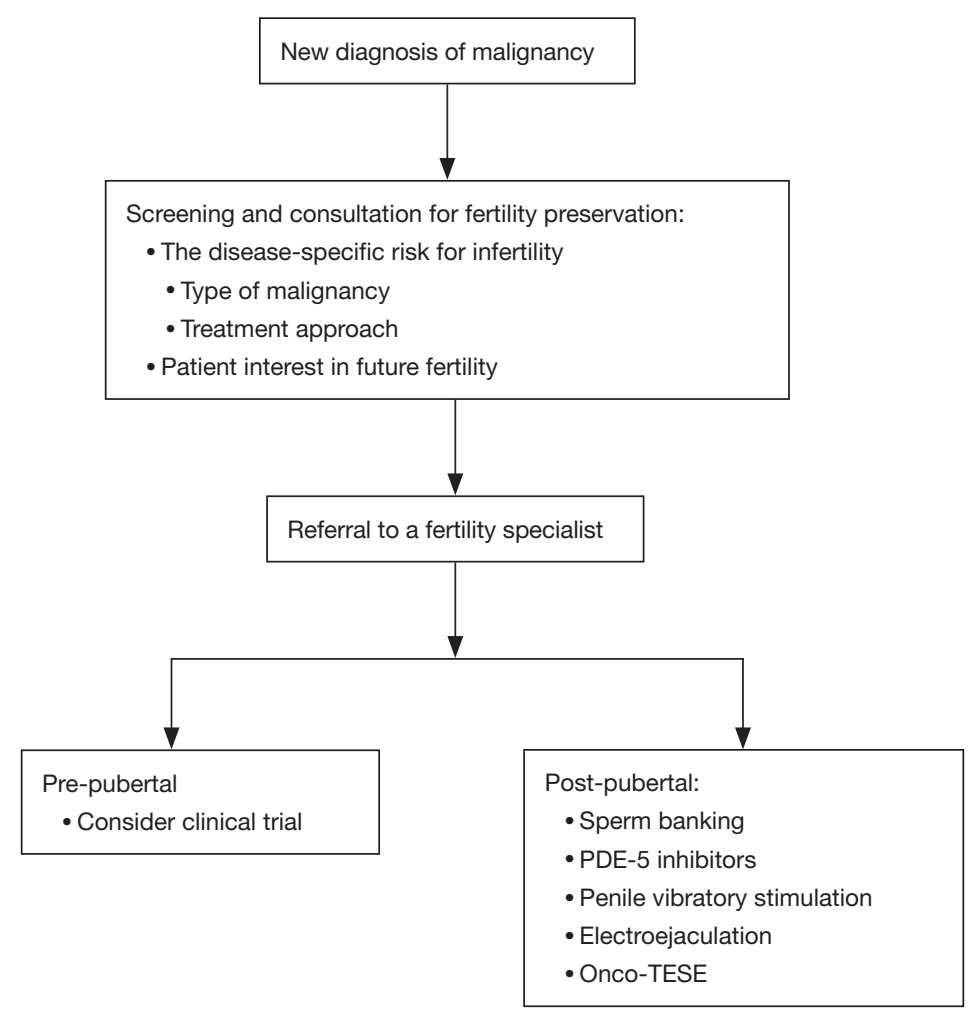

Figure 1 Approach to fertility preservation in the male cancer patient with a new diagnosis of malignancy (adapted with permission from Halpern et al., "Guideline based approach to male fertility preservation", Urologic Oncology 2019). PDE, phosphodiesterase; TESE, testicular sperm extraction.

reasonable cycle fecundity of $8.7 \%$ and $37.2 \%$ using intrauterine insemination (IUI) and IVF, respectively (78). Multiple studies have also demonstrated success using EEJ in the adolescent cancer population with sperm retrieval rates ranging from $45 \%$ to $60 \%(79,80)$. In contrast to PVS, EEJ is invasive and painful. While patients with spinal cord injury who lack sensation below the waist can undergo EEJ in the ambulatory setting without an anesthetic, most men will require anesthesia (81).

Despite the therapies above, a substantial proportion of men pursuing fertility preservation will remain azoospermic. Lass et al. reported $13.8 \%$ of cancer patients were azoospermic at the time of attempted sperm cryopreservation (82). These patients should be offered oncological testicular sperm extraction (oncoTESE) before initiation of any cancer treatment regimen. Initially coined by Schrader et al. in 2003, onco-TESE refers to the technique of testicular sperm extraction (TESE) when employed in the setting of malignancy, either using a conventional approach or a microdissection approach $(83,84)$. Like conventional TESE, onco-TESE carries a small but significant risk of complications such as hematoma, testicular fibrosis, hypogonadism, and even testicular loss (85). In a small series of 6 patients with either azoospermia, cryptozoospermia, or severe oligozoospermia who underwent onco-TESE, sperm was retrieved in $4(67 \%)$ patients (86). In the setting of testicular cancer, oncoTESE can be performed on the orchiectomy specimen ex vivo ("back table" onco-TESE), thereby sparing the morbidity of operation on the contralateral testis (87). For azoospermic men with a new cancer diagnosis, oncoTESE offers the best chance for future fertility, as many of these men will remain azoospermic after the completion of chemotherapy (86).

There is no consensus about monitoring of fertility after cancer. We recommend routine follow-up with repeat semen analysis at 1- and 2-years following completion of gonadotoxic therapy. Even in men with the return of sperm to the ejaculate sooner, we recommend waiting at least 1-2 years following gonadotoxic therapy before trying to conceive due to concerns about potential harm to offspring conceived during this interval. It is critical that providers 
discuss the importance of follow-up at the time of initial fertility preservation, as a large proportion $(36 \%)$ of men are lost to follow-up after completion of therapy (88).

\section{Fertility preservation in the pediatric patient}

Long-term longitudinal studies have demonstrated that men treated for childhood cancer are at significantly increased risk of infertility (89-91). There is great heterogeneity in the types of pediatric malignancy and treatments, which, along with patient age and pubertal state, can variably affect fertility potential. Unfortunately, gonadal shielding during radiation therapy and other preemptive maneuvers have been unsuccessful in consistently protecting future fertility (92).

The most important aspect in the management of pediatric fertility preservation is the determination of pubertal status with a detailed history and physical examination. It is imperative to discern whether the patient has experienced sexual thoughts, nocturnal emission, or masturbation, as this will inform his ability to supply a semen specimen. Physical examination with careful attention to secondary sex characteristics and Tanner staging can also provide insight into the pubertal state.

For peripubertal and post-pubertal adolescents, the goldstandard for fertility preservation is still cryopreservation of an ejaculated semen sample. Feasibility of cryopreservation of an ejaculated semen sample has been demonstrated in patients as young as 11 years, and for this reason, age alone should not be utilized as a determinant of pubertal state $(93,94)$. Peripubertal or post-pubertal males who are unable to supply a semen sample may be candidates for the therapies, including PVS, EEJ, and onco-TESE. Regardless of the mode of specimen collection, patients and their families should be counseled about the need for IVF with any future use of cryopreserved specimen.

Fertility preservation in prepubertal patients is still strictly experimental. Prepubertal patients do not have mature gametes or active spermatogenesis, and as such, they are not candidates for traditional cryopreservation (95). Several alternative experimental approaches have been undertaken to preserve fertility in prepubertal cancer patients. Spermatogonial stem cell transplantation has been described in multiple animal models, and more recent efforts have focused upon cryopreservation of testicular tissue with the goal of future culture and autologous grafting of spermatogonial stem cells. Others have explored the use of decellularized testicles or synthetic bioscaffolds for spermatogonial stem cell culture in attempts to cultivate viable testicular tissue in vitro $(96,97)$. Induction of spermatogonial stem cells from embryonic or induced pluripotent stem cells is yet another potential pathway (98).

Most recently, a breakthrough in autologous grafting of testicular tissue demonstrated great promise for future clinical application. Fayomi et al. harvested and cryopreserved testicular tissue from prepubertal rhesus macaques monkeys, which resulted in castration (99). Autologous grafting of cryopreserved tissue beneath either the back skin or scrotal skin of the castrated monkeys yielded testosterone production and complete spermatogenesis within 1 year. Using ART, graft-generated sperm successfully fertilized oocytes resulting in the birth of a healthy female. This exciting proof of concept might pave the way for future human studies of testicular tissue cryopreservation in prepubertal children.

\section{Barriers to fertility preservation}

There are numerous potential barriers to fertility preservation in both the adult and pediatric cancer patient. These are broad in scope and include patient and provider knowledge gaps, patient and provider discomfort, financial, logistical, and cultural barriers.

The most critical barrier to fertility preservation is the first consultation. A new cancer diagnosis is often accompanied by stress, anxiety, and a desire to start treatment at once. However, the narrow window before initiation of therapy is critical for fertility preservation, as even a single oncologic intervention may transiently or permanently impair fertility (56). Nonetheless, Schover et al. found that only $60 \%$ of adult men recall being informed about infertility as a possible side effect of their treatment and only $51 \%$ were offered sperm banking before therapy (8). Likewise, Quinn et al. reported that cancer patients of childbearing age were referred to reproductive endocrinologists less than half of the time (100). Physicians, nurses, and social workers alike may be unfamiliar or uncomfortable discussing fertility preservation with the newly diagnosed cancer patient, which may account for low referral rates (100-104). Oncologists are even less likely to refer patients for cryopreservation in the setting of aggressive cancer with a poor prognosis $(8,105)$. Since a physician's recommendation is an important predictor of fertility preservation, changing physician practice patterns, and closing knowledge gaps are essential to increasing 
utilization $(8,106)$.

Logistical considerations can severely limit access to fertility preservation in the acute setting. Many healthcare settings may not have a reproductive urologist on-site or even on-call for urgent consultation. Likewise, clinical facilities may not have the laboratory capabilities to perform sperm cryopreservation or even basic semen analysis $(107,108)$.

Even when appropriate counseling, referral, and resources are provided, financial considerations may impede fertility preservation $(102,103)$. Oncologists tend to overestimate the costs of cryopreservation, and $51 \%$ of oncologists believe out-of-pocket costs would prohibit most men from choosing this option $(8,109)$. However, a recent survey found that costs were not an important consideration for patients when deciding to cryopreserve sperm (110). Furthermore, while sperm cryopreservation can be expensive with estimated upfront and 3-year storage total fees approaching $\$ 1,500$, new legislation in multiple states has mandated insurance coverage for fertility preservation, thereby alleviating the financial burden $(57,111,112)$.

Cultural and religious beliefs may also impede fertility preservation. For example, masturbation is prohibited by certain religions including Roman Catholicism and Judaism, which can hinder the ability to obtain samples for cryopreservation (113). Likewise, certain religions do not support ART, thereby minimizing the utility of cryopreservation as the future use of sperm is severely restricted. Providers should explore these cultural and religious beliefs with patients during the first consultation to integrate these concerns into the plan of care.

Pediatric cancer patients present unique challenges when it comes to fertility preservation. Clinicians and parents alike may feel uncomfortable discussing sexual health and fertility with pediatric patients. Ethical considerations of consent, assent, and adherence to principles of beneficence, nonmaleficence, justice, and autonomy can manifest in many scenarios in both the prepubertal and post-pubertal patient (95).

\section{Role of a formal fertility preservation program}

A formal fertility preservation program can help to overcome the barriers above and optimize use of fertility preservation. Such a program should assemble a multidisciplinary team, including oncologists, urologists, reproductive endocrinologists, geneticists, mental health professionals, nurses, and social workers. Formal training programs and educational initiatives can improve the knowledge base of the team members and increase physician awareness throughout the institution (114). Online resources and patient navigators, available in multiple languages, can increase patient access.

Implementation of a fertility preservation program leads to improved outcomes. Sheth et al. reported an increase in the number of male cancer patients receiving fertility preservation consultation and pursuing sperm cryopreservation by 2.4- and 2.7-fold, respectively (114). Likewise, Lopategui et al. found a six-fold increase in fertility preservation after the establishment of an oncofertility team (115).

\section{Conclusions}

Cancer and its treatments can threaten fertility through a variety of mechanisms. There are many options for fertility preservation in male adult and pediatric cancer patients, and exciting, innovative approaches are on the horizon. Timely consultation, well-trained experts, and a formal fertility preservation program can help to overcome barriers and ensure that patients perfect their future fertility potential.

\section{Acknowledgments}

Funding: None.

\section{Footnote}

Provenance and Peer Review: This article was commissioned by the Guest Editors (Larry I. Lipshultz, Alexander W. Pastuszak) for the focused issue "Contemporary Issues and Controversies in Men's Health" published in Translational Andrology and Urology. The article was sent for external peer review organized by the Guest Editors and the editorial office.

Conflicts of Interest: The focused issue "Contemporary Issues and Controversies in Men's Health" was commissioned by the editorial office without any funding or sponsorship. The authors have no conflicts of interest to declare.

Ethical Statement: The authors are accountable for all aspects of the work in ensuring that questions related to the accuracy or integrity of any part of the work are appropriately investigated and resolved. 
Open Access Statement: This is an Open Access article distributed in accordance with the Creative Commons Attribution-NonCommercial-NoDerivs 4.0 International License (CC BY-NC-ND 4.0), which permits the noncommercial replication and distribution of the article with the strict proviso that no changes or edits are made and the original work is properly cited (including links to both the formal publication through the relevant DOI and the license). See: https://creativecommons.org/licenses/by-nc$\mathrm{nd} / 4.0 \%$.

\section{References}

1. Siegel RL, Miller KD, Jemal A. Cancer statistics, 2016. CA Cancer J Clin 2016;66:7-30.

2. Lambertini M, Del Mastro L, Pescio MC, et al. Cancer and fertility preservation: international recommendations from an expert meeting. BMC Med 2016;14:1.

3. Johnson JA, Tough S, Sogc Genetics C. Delayed childbearing. J Obstet Gynaecol Can 2012;34:80-93.

4. Lehmann V, Keim MC, Nahata L, et al. Fertilityrelated knowledge and reproductive goals in childhood cancer survivors: short communication. Hum Reprod 2017;32:2250-53.

5. Hartmann JT, Albrecht C, Schmoll HJ, et al. Long-term effects on sexual function and fertility after treatment of testicular cancer. Br J Cancer 1999;80:801-7.

6. Schover LR, Rybicki LA, Martin BA, et al. Having children after cancer. A pilot survey of survivors' attitudes and experiences. Cancer 1999;86:697-709.

7. Partridge AH, Gelber S, Peppercorn J, et al. Web-based survey of fertility issues in young women with breast cancer. J Clin Oncol 2004;22:4174-83.

8. Schover LR, Brey K, Lichtin A, et al. Knowledge and experience regarding cancer, infertility, and sperm banking in younger male survivors. J Clin Oncol 2002;20:1880-9.

9. Carter J, Rowland K, Chi D, et al. Gynecologic cancer treatment and the impact of cancer-related infertility. Gynecol Oncol 2005;97:90-5.

10. Letourneau JM, Ebbel EE, Katz PP, et al. Pretreatment fertility counseling and fertility preservation improve quality of life in reproductive age women with cancer. Cancer 2012;118:1710-7.

11. Miller KD, Siegel RL, Lin CC, et al. Cancer treatment and survivorship statistics, 2016. CA Cancer J Clin 2016;66:271-89.

12. Carroll PR, Whitmore WF Jr, Herr HW, et al. Endocrine and exocrine profiles of men with testicular tumors before orchiectomy. J Urol 1987;137:420-3.

13. de Bruin D, de Jong IJ, Arts EG, et al. Semen quality in men with disseminated testicular cancer: relation with human chorionic gonadotropin beta-subunit and pituitary gonadal hormones. Fertil Steril 2009;91:2481-6.

14. Hansen PV, Trykker H, Andersen J, et al. Germ cell function and hormonal status in patients with testicular cancer. Cancer 1989;64:956-61.

15. Yazama F, Tai A. Unexpected role of alpha-fetoprotein in spermatogenesis. PLoS One 2011;6:e19387.

16. Parker C, Milosevic M, Panzarella T, et al. The prognostic significance of the tumour infiltrating lymphocyte count in stage I testicular seminoma managed by surveillance. Eur J Cancer 2002;38:2014-9.

17. Theas MS, Rival C, Jarazo-Dietrich S, et al. Tumour necrosis factor-alpha released by testicular macrophages induces apoptosis of germ cells in autoimmune orchitis. Hum Reprod 2008;23:1865-72.

18. Rival C, Theas MS, Guazzone VA, et al. Interleukin-6 and IL-6 receptor cell expression in testis of rats with autoimmune orchitis. J Reprod Immunol 2006;70:43-58.

19. Wong EW, Mruk DD, Lee WM, et al. Regulation of blood-testis barrier dynamics by TGF-beta3 is a Cdc42dependent protein trafficking event. Proc Natl Acad Sci U S A 2010;107:11399-404.

20. Martínez P, Proverbio F, Camejo MI. Sperm lipid peroxidation and pro-inflammatory cytokines. Asian J Androl 2007;9:102-7.

21. Guazzieri S, Lembo A, Ferro G, et al. Sperm antibodies and infertility in patients with testicular cancer. Urology 1985;26:139-42.

22. Paoli D, Gilio B, Piroli E, et al. Testicular tumors as a possible cause of antisperm autoimmune response. Fertil Steril 2009;91:414-9.

23. Lerchbaum E, Obermayer-Pietsch B. Vitamin D and fertility: a systematic review. Eur J Endocrinol 2012;166:765-78.

24. Inui A. Cancer anorexia-cachexia syndrome: are neuropeptides the key? Cancer Res 1999;59:4493-501.

25. Campos CA, Bowen AJ, Han S, et al. Cancer-induced anorexia and malaise are mediated by CGRP neurons in the parabrachial nucleus. Nat Neurosci 2017;20:934-42.

26. Carlsen E, Andersson AM, Petersen JH, et al. History of febrile illness and variation in semen quality. Hum Reprod 2003;18:2089-92.

27. Marmor D, Elefant E, Dauchez C, et al. Semen analysis in Hodgkin's disease before the onset of treatment. Cancer 1986;57:1986-7. 
28. Viviani S, Ragni G, Santoro A, et al. Testicular dysfunction in Hodgkin's disease before and after treatment. Eur J Cancer 1991;27:1389-92.

29. Bhongade MB, Prasad S, Jiloha RC, et al. Effect of psychological stress on fertility hormones and seminal quality in male partners of infertile couples. Andrologia 2015;47:336-42.

30. Pallotti F, Petrozzi A, Cargnelutti F, et al. Long-Term Follow Up of the Erectile Function of Testicular Cancer Survivors. Front Endocrinol (Lausanne) 2019;10:196.

31. Stanton AM, Handy AB, Meston CM. Sexual function in adolescents and young adults diagnosed with cancer: A systematic review. J Cancer Surviv 2018;12:47-63.

32. Byrne J, Mulvihill JJ, Myers MH, et al. Effects of treatment on fertility in long-term survivors of childhood or adolescent cancer. N Engl J Med 1987;317:1315-21.

33. Buchanan JD, Fairley KF, Barrie JU. Return of spermatogenesis after stopping cyclophosphamide therapy. Lancet 1975;2:156-7.

34. Socié G, Salooja N, Cohen A, et al. Nonmalignant late effects after allogeneic stem cell transplantation. Blood 2003;101:3373-85.

35. Aslam I, Fishel S, Moore H, et al. Fertility preservation of boys undergoing anti-cancer therapy: a review of the existing situation and prospects for the future. Hum Reprod 2000;15:2154-9.

36. Green DM, Liu W, Kutteh WH, et al. Cumulative alkylating agent exposure and semen parameters in adult survivors of childhood cancer: a report from the St Jude Lifetime Cohort Study. Lancet Oncol 2014;15:1215-23.

37. Petersen PM, Hansen SW, Giwercman A, et al. Dosedependent impairment of testicular function in patients treated with cisplatin-based chemotherapy for germ cell cancer. Ann Oncol 1994;5:355-8.

38. Petersen PM, Giwercman A, Skakkebaek NE, et al. Gonadal function in men with testicular cancer. Semin Oncol 1998;25:224-33.

39. Howell SJ, Shalet SM. Spermatogenesis after cancer treatment: damage and recovery. J Natl Cancer Inst Monogr 2005;(34):12-7.

40. Brydøy M, Fossa SD, Klepp O, et al. Paternity and testicular function among testicular cancer survivors treated with two to four cycles of cisplatin-based chemotherapy. Eur Urol 2010;58:134-40.

41. Choudhury RC, Misra S, Jagdale MB, et al. Induction and transmission of cytogenetic toxic effects of 5-fluorouracil in male germline cells of Swiss mice. J Exp Clin Cancer Res 2002;21:277-82.
42. Thomson AB, Campbell AJ, Irvine DC, et al. Semen quality and spermatozoal DNA integrity in survivors of childhood cancer: a case-control study. Lancet 2002;360:361-7.

43. Aubier F, Flamant F, Brauner R, et al. Male gonadal function after chemotherapy for solid tumors in childhood. J Clin Oncol 1989;7:304-9.

44. Sjöblom T, Parvinen M, Lahdetie J. Stage-specific DNA synthesis of rat spermatogenesis as an indicator of genotoxic effects of vinblastine, mitomycin $\mathrm{C}$ and ionizing radiation on rat spermatogonia and spermatocytes. Mutat Res 1995;331:181-90.

45. Foster RS, Bennett R, Bihrle R, et al. A preliminary report: postoperative fertility assessment in nerve-sparing RPLND patients. Eur Urol 1993;23:165-7; discussion 168.

46. Beck SD, Bey AL, Bihrle R, et al. Ejaculatory status and fertility rates after primary retroperitoneal lymph node dissection. J Urol 2010;184:2078-80.

47. Lange MM, van de Velde CJ. Urinary and sexual dysfunction after rectal cancer treatment. Nat Rev Urol 2011;8:51-7.

48. Eveno C, Lamblin A, Mariette C, et al. Sexual and urinary dysfunction after proctectomy for rectal cancer. J Visc Surg 2010;147:e21-30.

49. Jacobsen KD, Ous S, Waehre H, et al. Ejaculation in testicular cancer patients after post-chemotherapy retroperitoneal lymph node dissection. Br J Cancer 1999;80:249-55.

50. Terrone C, Cracco C, Scarpa RM, et al. Supra-ampullar cystectomy with preservation of sexual function and ileal orthotopic reservoir for bladder tumor: twenty years of experience. Eur Urol 2004;46:264-9; discussion 269-70.

51. Heyn R, Raney RB Jr, Hays DM, et al. Late effects of therapy in patients with paratesticular rhabdomyosarcoma. Intergroup Rhabdomyosarcoma Study Committee. J Clin Oncol 1992;10:614-23.

52. T Rague J, Varda BK, Wagner AA, et al. Delayed Return of Ejaculatory Function in Adolescent Males Treated With Retroperitoneal Lymph Node Dissection and Adjuvant Therapy for Paratesticular Rhabdomyosarcoma. Urology 2019;124:254-6.

53. Maurer CA, Z'Graggen K, Renzulli P, et al. Total mesorectal excision preserves male genital function compared with conventional rectal cancer surgery. Br J Surg 2001;88:1501-5.

54. Lim RS, Yang TX, Chua TC. Postoperative bladder and sexual function in patients undergoing surgery for rectal cancer: a systematic review and meta-analysis of 
laparoscopic versus open resection of rectal cancer. Tech Coloproctol 2014;18:993-1002.

55. Jacobsen KD, Theodorsen L, Fossa SD. Spermatogenesis after unilateral orchiectomy for testicular cancer in patients following surveillance policy. J Urol 2001;165:93-6.

56. Oktay K, Harvey BE, Partridge AH, et al. Fertility Preservation in Patients With Cancer: ASCO Clinical Practice Guideline Update. J Clin Oncol 2018;36:1994-2001.

57. Lee SJ, Schover LR, Partridge AH, et al. American Society of Clinical Oncology recommendations on fertility preservation in cancer patients. J Clin Oncol 2006;24:2917-31.

58. Coccia PF, Altman J, Bhatia S, et al. Adolescent and young adult oncology. Clinical practice guidelines in oncology. J Natl Compr Canc Netw 2012;10:1112-50.

59. Coccia PF, Pappo AS, Altman J, et al. Adolescent and young adult oncology, version 2.2014. J Natl Compr Canc Netw 2014;12:21-32; quiz 32.

60. Peccatori FA, Azim HA Jr, Orecchia R, et al. Cancer, pregnancy and fertility: ESMO Clinical Practice Guidelines for diagnosis, treatment and follow-up. Ann Oncol 2013;24 Suppl 6:vi160-70.

61. Fallat ME, Hutter J, American Academy of Pediatrics Committee on B, et al. Preservation of fertility in pediatric and adolescent patients with cancer. Pediatrics 2008;121:e1461-9.

62. The Ethics Committee of the American Society for Reproductive Medicine. Fertility preservation and reproduction in patients facing gonadotoxic therapies: a committee opinion. Fertil Steril 2013;100:1224-31.

63. The Ethics Committee of the American Society for Reproductive Medicine. Fertility preservation and reproduction in cancer patients. Fertil Steril 2005;83:1622-8.

64. Ethics Committee of the American Society for Reproductive Medicine. Electronic address: ASRM@asrm. org. Fertility preservation and reproduction in patients facing gonadotoxic therapies: an Ethics Committee opinion. Fertil Steril 2018;110:380-6.

65. Anger JT, Gilbert BR, Goldstein M. Cryopreservation of sperm: indications, methods and results. J Urol 2003;170:1079-84.

66. Donnelly ET, McClure N, Lewis SE. Cryopreservation of human semen and prepared sperm: effects on motility parameters and DNA integrity. Fertil Steril 2001;76:892-900.
67. Kuczy ski W, Dhont M, Grygoruk C, et al. The outcome of intracytoplasmic injection of fresh and cryopreserved ejaculated spermatozoa--a prospective randomized study. Hum Reprod 2001;16:2109-13.

68. Depalo R, Falagario D, Masciandaro P, et al. Fertility preservation in males with cancer: 16-year monocentric experience of sperm banking and post-thaw reproductive outcomes. Ther Adv Med Oncol 2016;8:412-20.

69. Esteves SC, Chan P. A systematic review of recent clinical practice guidelines and best practice statements for the evaluation of the infertile male. Int Urol Nephrol 2015;47:1441-56.

70. Voznesensky M, Annam K, Kreder KJ. Understanding and Managing Erectile Dysfunction in Patients Treated for Cancer. J Oncol Pract 2016;12:297-304.

71. Burnett AL, Nehra A, Breau RH, et al. Erectile Dysfunction: AUA Guideline. J Urol 2018;200:633-41.

72. Mehta A, Sigman M. Management of the dry ejaculate: a systematic review of aspermia and retrograde ejaculation. Fertil Steril 2015;104:1074-81.

73. Shoshany O, Abhyankar N, Elyaguov J, et al. Efficacy of treatment with pseudoephedrine in men with retrograde ejaculation. Andrology 2017;5:744-8.

74. Brassesco M, Viscasillas P, Burrel L, et al. Sperm recuperation and cervical insemination in retrograde ejaculation. Fertil Steril 1988;49:923-5.

75. Silva PD, Larson KM, Van Every MJ, et al. Successful treatment of retrograde ejaculation with sperm recovered from bladder washings. A report of two cases. J Reprod Med 2000;45:957-60.

76. Castle SM, Jenkins LC, Ibrahim E, et al. Safety and efficacy of a new device for inducing ejaculation in men with spinal cord injuries. Spinal Cord 2014;52 Suppl 2:S27-9.

77. Kafetsoulis A, Brackett NL, Ibrahim E, et al. Current trends in the treatment of infertility in men with spinal cord injury. Fertil Steril 2006;86:781-9.

78. Ohl DA, Wolf LJ, Menge AC, et al. Electroejaculation and assisted reproductive technologies in the treatment of anejaculatory infertility. Fertil Steril 2001;76:1249-55.

79. Berookhim BM, Mulhall JP. Outcomes of operative sperm retrieval strategies for fertility preservation among males scheduled to undergo cancer treatment. Fertil Steril 2014;101:805-11.

80. Adank MC, van Dorp W, Smit M, et al. Electroejaculation as a method of fertility preservation in boys diagnosed with cancer: a single-center experience and review of the literature. Fertil Steril 2014;102:199-205.e1. 
81. Revenig L, Leung A, Hsiao W. Ejaculatory physiology and pathophysiology: assessment and treatment in male infertility. Transl Androl Urol 2014;3:41-9.

82. Lass A, Akagbosu F, Abusheikha N, et al. A programme of semen cryopreservation for patients with malignant disease in a tertiary infertility centre: lessons from 8 years' experience. Hum Reprod 1998;13:3256-61.

83. Schrader M, Muller M, Sofikitis N, et al. "Oncotese": testicular sperm extraction in azoospermic cancer patients before chemotherapy-new guidelines? Urology 2003;61:421-5.

84. Schlegel PN. Testicular sperm extraction: microdissection improves sperm yield with minimal tissue excision. Hum Reprod 1999;14:131-5.

85. Eliveld J, van Wely M, Meissner A, et al. The risk of TESE-induced hypogonadism: a systematic review and meta-analysis. Hum Reprod Update 2018;24:442-54.

86. Furuhashi K, Ishikawa T, Hashimoto H, et al. Oncotesticular sperm extraction: testicular sperm extraction in azoospermic and very severely oligozoospermic cancer patients. Andrologia 2013;45:107-10.

87. Luján S, Guzman-Ordaz D, Rogel R, et al. ONCOTESE: Obtaining spermatozoa after radical orchiectomy for testicular tumour and azoospermia. Actas Urol Esp 2016;40:64-7.

88. Pacey AA, Merrick H, Arden-Close E, et al. Monitoring fertility (semen analysis) by cancer survivors who banked sperm prior to cancer treatment. Hum Reprod 2012;27:3132-9.

89. Green DM, Kawashima T, Stovall M, et al. Fertility of male survivors of childhood cancer: a report from the Childhood Cancer Survivor Study. J Clin Oncol 2010;28:332-9.

90. Wasilewski-Masker K, Seidel KD, Leisenring W, et al. Male infertility in long-term survivors of pediatric cancer: a report from the childhood cancer survivor study. J Cancer Surviv 2014;8:437-47.

91. Barton SE, Najita JS, Ginsburg ES, et al. Infertility, infertility treatment, and achievement of pregnancy in female survivors of childhood cancer: a report from the Childhood Cancer Survivor Study cohort. Lancet Oncol 2013;14:873-81.

92. Ishiguro H, Yasuda Y, Tomita Y, et al. Gonadal shielding to irradiation is effective in protecting testicular growth and function in long-term survivors of bone marrow transplantation during childhood or adolescence. Bone Marrow Transplant 2007;39:483-90.

93. Keene DJ, Sajjad Y, Makin G, et al. Sperm banking in the
United Kingdom is feasible in patients 13 years old or older with cancer. J Urol 2012;188:594-7.

94. Halpern JA, Thirumavalavan N, Kohn TP, et al. Distribution of Semen Parameters Among Adolescent Males Undergoing Fertility Preservation in a Multicenter International Cohort. Urology 2019;127:119-23.

95. Johnson EK, Finlayson C, Rowell EE, et al. Fertility Preservation for Pediatric Patients: Current State and Future Possibilities. J Urol 2017;198:186-94.

96. Shams A, Eslahi N, Movahedin M, et al. Future of Spermatogonial Stem Cell Culture: Application of Nanofiber Scaffolds. Curr Stem Cell Res Ther 2017;12:544-53.

97. Komeya M, Ogawa T. Spermatogonial stem cells: Progress and prospects. Asian J Androl 2015;17:771-5.

98. Hayashi K, Ohta H, Kurimoto K, et al. Reconstitution of the mouse germ cell specification pathway in culture by pluripotent stem cells. Cell 2011;146:519-32.

99. Fayomi AP, Peters K, Sukhwani M, et al. Autologous grafting of cryopreserved prepubertal rhesus testis produces sperm and offspring. Science 2019;363:1314-9.

100. Quinn GP, Vadaparampil ST, King L, et al. Impact of physicians' personal discomfort and patient prognosis on discussion of fertility preservation with young cancer patients. Patient Educ Couns 2009;77:338-43.

101.Köhler TS, Kondapalli LA, Shah A, et al. Results from the survey for preservation of adolescent reproduction (SPARE) study: gender disparity in delivery of fertility preservation message to adolescents with cancer. J Assist Reprod Genet 2011;28:269-77.

102. Quinn GP, Vadaparampil ST, Bell-Ellison BA, et al. Patient-physician communication barriers regarding fertility preservation among newly diagnosed cancer patients. Soc Sci Med 2008;66:784-9.

103.King L, Quinn GP, Vadaparampil ST, et al. Oncology nurses' perceptions of barriers to discussion of fertility preservation with patients with cancer. Clin J Oncol Nurs 2008; 12:467-76.

104. Quinn GP, Vadaparampil ST, Gwede CK, et al. Discussion of fertility preservation with newly diagnosed patients: oncologists' views. J Cancer Surviv 2007;1:146-55.

105. Roopnarinesingh R, Keane D, Harrison R. Detecting mood disorders in men diagnosed with cancer who seek semen cryopreservation: a chance to improve service. Ir Med J 2003;96:104, 106-7.

106.Fisher JR, Hammarberg K, Baker HW. Assisted conception is a risk factor for postnatal mood disturbance and early parenting difficulties. Fertil Steril 
2005;84:426-30.

107. Clayman ML, Harper MM, Quinn GP, et al. Oncofertility resources at NCI-designated comprehensive cancer centers. J Natl Compr Canc Netw 2013;11:1504-9.

108. Bowman-Curci M, Quinn GP, Reinecke J, et al.

Comparing fertility preservation resources and policies between NCCN member and non-member institutions. Support Care Cancer 2019;27:2125-9.

109.Zapzalka DM, Redmon JB, Pryor JL. A survey of oncologists regarding sperm cryopreservation and assisted reproductive techniques for male cancer patients. Cancer 1999;86:1812-7.

110. Scott-Trainer J. The role of a patient navigator in fertility preservation. Cancer Treat Res 2010;156:469-70.

111. Alliance for Fertility Preservation. Families, Advocates Praise Passage of IVF \& Fertility Preservation Insurance

Cite this article as: Halpern JA, Das A, Faw CA, Brannigan RE. Oncofertility in adult and pediatric populations: options and barriers. Transl Androl Urol 2020;9(Suppl 2):S227-S238. doi: $10.21037 /$ tau.2019.09.27
Reforms in NY State Budget. 2019:1.

112. Stoner K. Cancer Patients Guaranteed Oncofertility Treatment Coverage Under New Illinois Law.

Northwestern Medicine, Aug 28, 2018.

113.Ramstein JJ, Halpern J, Gadzinski AJ, et al. Ethical, moral, and theological insights into advances in male pediatric and adolescent fertility preservation. Andrology 2017;5:631-39.

114. Sheth KR, Sharma V, Helfand BT, et al. Improved fertility preservation care for male patients with cancer after establishment of formalized oncofertility program. J Urol 2012;187:979-86.

115.Lopategui DM, Ibrahim E, Aballa TC, et al. Effect of a formal oncofertility program on fertility preservation ratesfirst year experience. Transl Androl Urol 2018;7:S271-5. 